\title{
Analytic approach to modeling of nano-dots growth on the grazing incidence mirror surface under FEL irradiation
}

\author{
I.V. Kozhevnikov*a ${ }^{\mathrm{a}}$, A.V. Buzmakov ${ }^{\mathrm{a}}$, F. Siewert ${ }^{\mathrm{b}}$, K. Tiedtke ${ }^{\mathrm{c}}$, M. Störmer ${ }^{\mathrm{d}}$, L. Samoylova ${ }^{\mathrm{e}}$, \\ H. Sinn ${ }^{\text {e }}$ \\ aShubnikov Institute of Crystallography of Federal Research Centre "Crystallography and Photonics"

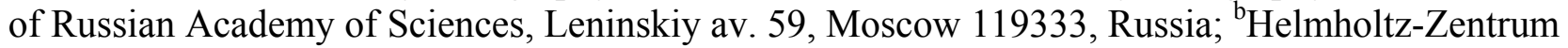 \\ Berlin für Materialien und Energie GmbH, Albert Einstein Str. 15, 12489 Berlin, Germany; \\ ${ }^{\mathrm{c}}$ Deutsches Elektronen-Synchrotron DESY, Notkestrasse 85, 22603 Hamburg, Germany; \\ ${ }^{\mathrm{d}}$ Helmholtz-Zentrum Geesthacht, Institute of Materials Research, Max-Planck-Str. 1, 21502 \\ Geesthacht, Germany; ${ }^{\mathrm{e}}$ European XFEL GmbH, Holzkoppel 4, 22869 Schenefeld, Germany
}

\begin{abstract}
Simple analytic equation is deduced to explain new physical phenomenon detected experimentally: growth of nano-dots (40-55 nm diameter, $8-13 \mathrm{~nm}$ height, $9.4 \mathrm{dots} / \mathrm{\mu m}^{2}$ surface density) on the grazing incidence mirror surface under the three years irradiation by the free electron laser FLASH (5-45 $\mathrm{nm}$ wavelength, 3 degrees grazing incidence angle). The growth model is based on the assumption that the growth of nano-dots is caused by polymerization of incoming hydrocarbon molecules under the action of incident photons directly or photoelectrons knocked out from a mirror surface. The key feature of our approach consists in that we take into account the radiation intensity variation nearby a mirror surface in an explicit form, because the polymerization probability is proportional to it. We demonstrate that the simple analytic approach allows to explain all phenomena observed in experiment and to predict new effects. In particular, we show that the nano-dots growth depends crucially on the grazing angle of incoming beam and its intensity: growth of nano-dots is observed in the limited from above and below intervals of the grazing angle and the radiation intensity. Decrease in the grazing angle by 1 degree only (from 3 to 2 degree) may result in a strong suppression of nanodots growth and their total disappearing. Similarly, decrease in the radiation intensity by several times (replacement of free electron laser by synchrotron) results also in disappearing of nano-dots growth.
\end{abstract}

Keywords: free electron lasers, nano-dots, FEL mirrors, interaction of FEL radiation with matter

\section{INTRODUCTION}

In the previous our paper [1] new physical phenomenon was experimentally discovered: growth of spikes (nano-dots) of the 30-60 nm diameter, $6-13 \mathrm{~nm}$ height, and $9.4 \mathrm{dots} / \mu \mathrm{m}^{2}$ surface density on the grazing incidence mirror surface under the three years irradiation by the free electron laser (FEL) FLASH (5-45 nm wavelength, 3 degrees grazing incidence angle) [2]. Inspection of another mirror operating at similar conditions, but at the 2 degrees grazing incidence angle demonstrated strong suppression of the spikes growth: nano-dots of only 3-4 nm height and $0.23 \mathrm{dots} / \mu \mathrm{m}^{2}$ surface density were observed.

The model suggested in [1] allowed us to explain the main features of the spikes growth under FEL irradiation. Our approach was based on the assumption that the growth of nano-dots is caused by polymerization or carbonization (cracking and subsequent strong chemical bonding) of incoming hydrocarbon molecules under the action of incident photons directly or photoelectrons knocked out from a mirror surface. Notice that there are manifold works (see, e.g. $[3,4]$ ), where a growth of carbonaceous layer on a surface of optical elements placed in synchrotron beamlines or in optical systems of extreme ultraviolet lithography is also explained by radiation or photoelectron induced carbonization. The main difference of our model consists in that we take into account the radiation intensity variation nearby a mirror surface in an explicit form.

*ivk@crys.ras.ru; phone +7 4991355100 
Evidently, the probability of radiation-induced cracking and subsequent polymerization of adsorbed hydrocarbon molecules is proportional to the field intensity (the photon flux density) $|E|^{2}$ on the surface. Similarly, the probability of polymerization under the action of photoelectrons is proportional to their density in the point of the molecule dropping on a surface, which, in its turn, is proportional to the radiation power absorbed in the matter, i.e., the value of the field intensity $|E|^{2}$ on the surface once again. If the grazing angle is small compared to the critical angle of the total external reflection (TER), the field intensity is low at the mirror surface, while it is increased in vacuum with increasing distance to the surface (Fig.1). Therefore, if there is a surface feature (e.g., a peak on a rough surface), the field intensity on its top is higher and, hence, probability of polymerization of incoming molecules increases resulting in a quicker growth of the feature compared to the growth of underlying surface. As a result, the positive feedback arises: the higher the feature on the surface is, the quicker its growth occurs and finally the feature appears as nano-dot or spike in an AFM image.

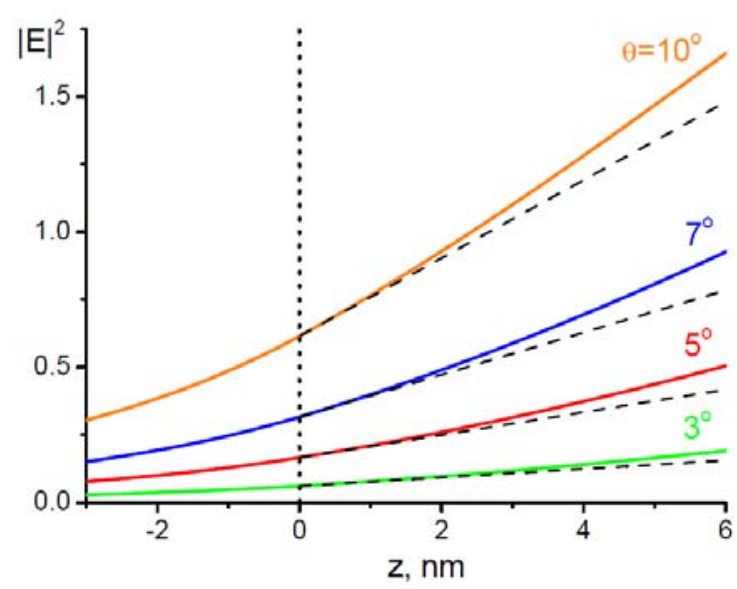

Fig. 1. Field intensity (at $\lambda=20 \mathrm{~nm}$ ) nearby ideally smooth carbon surface placed at $\mathrm{z}=0$ for different grazing angles $\theta$ of the incident beam, the value of $\theta$ being indicated in the graph. The amplitude of the incident wave was set to unity for definiteness. Z-axis is directed into vacuum. Vertical dotted line at $z=0$ corresponds to the position of the reflecting surface. Straight dashed lines show the field intensity in vacuum after linearization with the use of Eq. (6).

The crucial factors responsible for the growth of nano-dots were theoretically demonstrated to be the incident peak intensity and the reflection angle of the beam. A reduction of the peak intensity by only several times (e.g., replacement of FEL beam by synchrotron radiation) as well as a decrease in the incident angle by just one degree (from 3 to 2 degrees) may result in a strong suppression of the nano-dots growth or even their total disappearing.

In the previous paper we analyzed the process of nano-dots growth using the vivid approach of F. Family and T. Viscek $[5,6]$, where the film growth was modeled by representation of incoming molecules flux as a set of small cubes falling randomly onto the surface and forming growing columns. In the present paper, we deduce very simple, while approximate, analytic expression describing qualitatively the nano-dots growth. New physical effects, which were not described in the previous our paper, are analyzed.

\section{ANALYTIC THEORY OF NANO-DOTS GROWTH UNDER FEL IRRADIATION}

Following [1] we assume that three and only three different events may occur after hydrocarbon molecule falls onto the surface. First, the molecule sticks at once to it due to polymerization under the action of photoelectrons with the probability $P_{\mathrm{pol}}$. Second, the molecule is reflected (desorbed) from the surface and comes back to vacuum with the probability $P_{\mathrm{des}}$. Finally, the molecule diffuses along the surface with the probability $P_{\mathrm{dif}}=1-P_{\mathrm{pol}}-P_{\mathrm{des}}$, whereupon it sticks to the surface due to polymerization. The experimental values of the probabilities $P_{\text {des }}$ and $P_{\text {dif }}$ are scarcely known, and their calculation from the first principles is a very complex problem, if is at all possible. Therefore the ratio $\xi \equiv P_{d e s} / P_{d i f}$ is considered as the free parameter of our model. 
Suppose that a surface relief in the moment $\tau$ in time is described by the function $z=h(\rho, \tau)$, where $\rho=x$ is a scalar in the case of $1+1$ dimensional surface and $\rho=(x, y)$ is a vector for $1+2$ dimensional surface. The probability of polymerization $P_{\text {pol }}$ is supposed to be proportional to the photoelectron density $Q(\rho, h)$ in the point $\rho$ of the molecule drop onto surface. In its turn, the density $Q$ is proportional to X-ray radiation absorption in a small volume of material nearby this point, i.e. $Q(\rho, h) \sim A^{2}\left|E_{0}(\rho, h)\right|^{2} \operatorname{Im} \varepsilon$, where $\varepsilon$ is the complex dielectric constant of matter, $A$ is the amplitude of the incident wave, and $E_{0}$ is the field function assuming the amplitude of a plane incident wave to equal to unity (see [1] for more details).

In addition, we assumed that the density of photoelectrons in the point, where the molecule dropped onto a top of the surface, is proportional to a number $N$ of molecules surrounding the dropped one. In other words, we supposed that the photoelectrons density is higher in a valley on a rough surface as compared with that on its peak. Basing on these assumptions we represented the probability of polymerization of the molecule dropped onto a top of the surface in the point $\rho$ as $P_{p o l}(\rho)=\eta N\left|E_{0}(h(\rho)-\bar{h})\right|^{2}$, where the second free parameter of the model $\eta$ is proportional to the field intensity $A^{2}$. To guarantee the polymerization probability lesser than unity we re-wrote the expression for $P_{p o l}$ as follow

$$
P_{p o l}(\rho)=1-\exp \left(-\eta N\left|E_{0}(h(\rho)-\bar{h})\right|^{2}\right)
$$

Instead of $\eta$ we will use below the value of the clearly understandable physical parameter, namely, the polymerization probability $P_{0}$ of the molecule falling onto a flat surface, when $h(\rho)=\bar{h}$ and the number of surrounding molecules $N \equiv N_{0}$ is equal to either $3(1+1$ dimensional surface $)$ or $9(1+2$ dimensional surface):

$$
P_{0}(\theta)=1-\exp \left(-N_{0} \eta|t(\theta)|^{2}\right)
$$

where $t$ is the field amplitude on a flat surface (amplitude transmittance determined by the Fresnel formula) and $\theta$ is the grazing incidence angle.

Let $I$ to be an incident flux of incoming hydrocarbon molecules (in particle $/ \mathrm{nm}^{2} / \mathrm{s}$ for $1+2$ dimensional surface). Then $d n=I d S d \tau$ molecules fall onto a small area $d S$ on a growing film surface during the short temporal interval $d \tau$. Taking into account that a part $P_{d e s}$ of molecules are desorbed and assuming $\Omega$ to be a volume occupied by a single molecule inside the film (in $\mathrm{nm}^{3}$ ) we find that the film volume is increased by $d V \equiv d S d h=I \Omega d S d \tau\left(1-P_{d e s}\right)$ and obtain the simplest equation describing the carbonaceous film growth with time

$$
\frac{d h(\rho, \tau)}{d \tau}=I \Omega\left[1-P_{d e s}(h-\bar{h})\right]
$$

where $h(\rho, \tau)$ describes a surface of the growing film, and $\bar{h}$ is the averaged layer thickness. The equation neglects the random nature of incident flux of molecules, assumes that it is constant both in space and in time, and thus describes the nano-dots growth averaged over different realizations of random flux. Moreover, the equation does not take into complete account the surface diffusion of incoming molecules, while the effect of diffusion is introduced via the relation $1-P_{d e s}=P_{p o l}+P_{d i f}$. The fact is that, according to the results obtained in [1], the nano-dots growth is clearly observed, if the surface diffusion appears only weakly. Using the free parameter of our model $\xi \equiv P_{d e s} / P_{d i f}$, we can express the probability of desorption via the probability of polymerization as $1-P_{d e s}(h-\bar{h})=\left[1+\xi P_{p o l}(h-\bar{h})\right] /(1+\xi)$.

In particular, an increase in the averaged film thickness is governed by the equation $d \bar{h}(\tau) / d \tau=I \Omega\left(1+\xi P_{0}\right) /(1+\xi)$, where the right side is independent of time. Then we immediately obtain the following equation describing a growth of nano-dots with the increasing carbonaceous film thickness $\bar{h}$ : 


$$
\frac{d(h-\bar{h})}{d \bar{h}}=\frac{\xi}{1+\xi P_{0}}\left[P_{p o l}(h-\bar{h})-P_{0}\right]
$$

The probabilities of polymerization $P_{0}$ and $P_{p o l}$ in Eq. (5) are determined by the intensity of incident radiation via Eq. (2), what is the key point of our approach. To find solution of Eq. (5) in an analytical form we suppose that the spikes height $h-\bar{h}$ is small enough, so that we can linearize the expression for the radiation intensity in vacuum nearby a surface as

$$
\left|E_{0}(h-\bar{h})\right|^{2} \approx|t|^{2}-4 \operatorname{Im} r \cdot k(h-\bar{h}) \sin \theta
$$

where $r(\operatorname{Im} r \leq 0)$ is the amplitude reflectance determined by the Fresnel formulas.

Next, the number of surrounding molecules $N$ in Eqs. (2), (5) depends on the point on a surface, where the given molecule falls, and it can vary from 1 to $7(1+1$ surface) or from 1 to 25 (1+2 surface). To solve Eq. (5) analytically we set the number of surrounding molecules to the same average value independently of the point, where molecule falls on a surface, i.e. put $N=N_{0}=\bar{N}$ in Eq. (5), where $\bar{N}=4$ or 13 for $1+1$ or $1+2$ dimensional surface, respectively. Then we obtain the following equation

$$
\frac{d(h-\bar{h})}{d \bar{h}}=\frac{\xi \mathrm{e}^{-\bar{N} \eta|t(\theta)|^{2}}}{1+\xi \mathrm{e}^{-\bar{N} \eta|t(\theta)|^{2}}}\left[1-\mathrm{e}^{4 \operatorname{Im} r(\theta) \cdot k(h-\bar{h}) \sin \theta}\right]
$$

whose solution can be easily found in an explicit analytical form:

$$
h(\rho)=\bar{h}+\frac{1}{k U(\theta)} \ln \left[1+\left(\mathrm{e}^{k U(\theta) h_{0}(\rho)}-1\right) \cdot \mathrm{e}^{k U(\theta) V(\theta) \bar{h}}\right]
$$

The functions $h_{0}(\rho)$ and $h(\rho)$ in Eq. (8) describe a virgin surface relief and that of a surface after irradiation, respectively, and the following dimensionless parameters were introduced to shorten Eq. (8):

$$
U(\theta)=-4 \bar{N} \eta \sin \theta \operatorname{Im} r(\theta) ; \quad V(\theta)=\frac{\xi \mathrm{e}^{-\bar{N} \eta|t(\theta)|^{2}}}{1+\xi\left(1-\mathrm{e}^{-\bar{N} \eta|t(\theta)|^{2}}\right)}
$$

The parameters $\bar{N}$ and $\eta$ are only appeared as the product $\bar{N} \eta$ in Eq. (9). Therefore, Eqs. (8)-(9) result in conclusion that a growth of $1+2$ dimensional carbonaceous film $(\bar{N}=13)$ occurs by the same manner as a growth of $1+1$ dimensional film ( $\bar{N}=4$ ), if the parameter $\eta$ is respectively renormalized to keep the constant product $\bar{N} \eta$.

\section{ANALYSIS OF SPIKES GROWTH USING ANALYTIC APPROACH}

In the previous our paper we established the following peculiarities of the spikes growth using numerical modeling:

- The spikes growth is observed, if the probability of the surface diffusion of incoming hydrocarbon molecules is not too high.

- If the incident radiation intensity is fixed, the spikes growth is only observed in a limited, from above and below, interval of the grazing angles of incident radiation.

- Decreasing the incident radiation intensity by several times may result in a total disappearing of the spikes growth.

Below we will demonstrate that all these features are described by the simple analytic theory (Eqs. (8)-(9)), which, in addition, will allow us to predict new effects not described in the previous paper. For definiteness, we set the radiation wavelength to $\lambda=20 \mathrm{~nm}$ and will analyze a growth of nano-dot on a smooth surface having the only parabolic feature of the $1.5 \mathrm{~nm}$ maximal height and the $100 \mathrm{~nm}$ length. The case of $1+1$ dimensional surface is considered, while according to Eqs. (8)-(9) the results remain the same for 1+2 dimensional surface, if the parameter $\eta$ is decreased by a factor of $14 / 3$. 
Dependence of the spike height $\Delta h$ on the grazing angle of incident radiation is shown in Fig. 2. Calculations were performed using Eq. (8) for different diffusion parameters $\xi$ and the fixed radiation flux density (parameter $\eta=1)($ Fig. $2 \mathrm{a}$ ) as well as for different radiation flux densities (parameters $\eta$ ) and the fixed diffusion parameter $\xi=6$ (Fig. $2 \mathrm{~b}$ ). When calculating, the averaged carbonaceous film thickness was set to the same value $\bar{h}=15 \mathrm{~nm}$. Figure 2 a demonstrates clearly that the nano-dots growth is well pronounced, if only the surface diffusion is weak, i.e. the parameter $\xi$ is large.
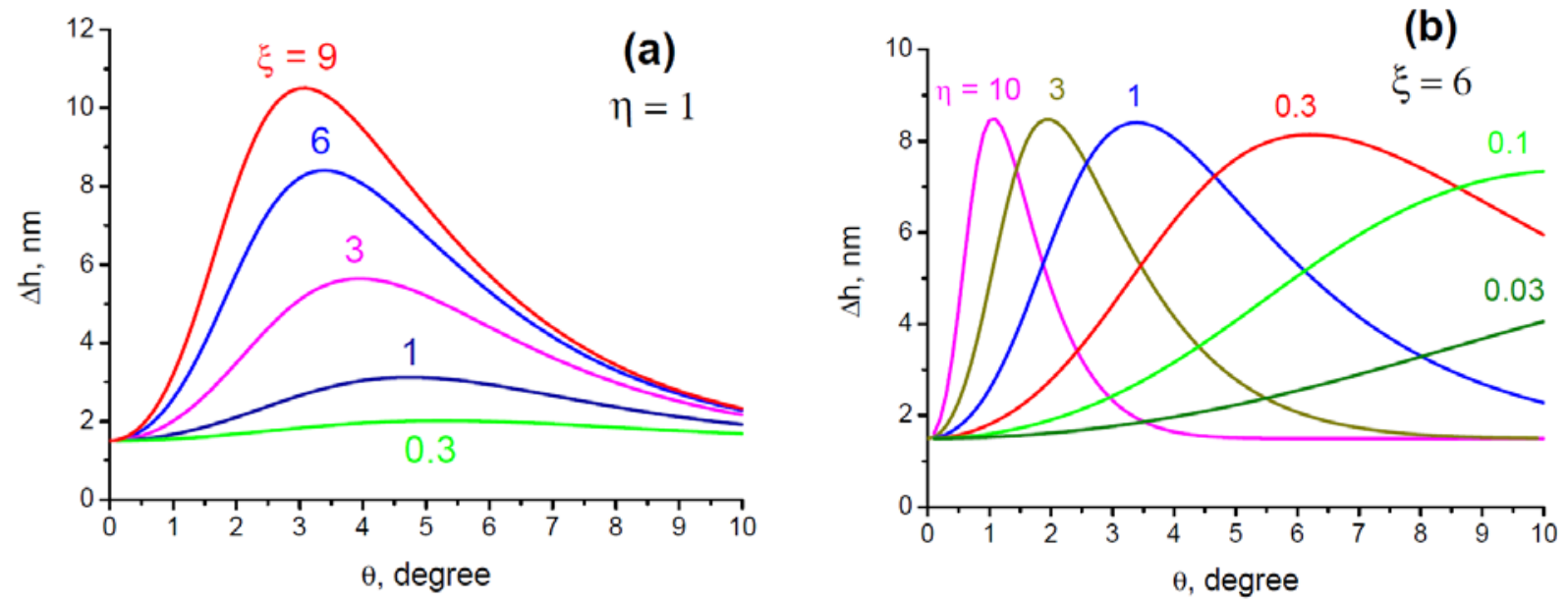

Fig. 2. Dependence of the spike height on the grazing angle of incident radiation $(\lambda=20 \mathrm{~nm})$. Calculations were performed for (a) different diffusion parameters $\xi$ and the fixed radiation flux density (parameter $\eta=1$ ); and (b) different radiation flux densities (parameters $\eta$ ) and the fixed diffusion parameter $\xi=6$. The feature height on a virgin substrate was $1.5 \mathrm{~nm}$ and the averaged carbonaceous film thickness is equal to $\bar{h}=15 \mathrm{~nm}$.

Figure $2 \mathrm{~b}$ demonstrates that the spike height achieves the maximal value at a certain angle depending on the field intensity on a surface. In particularly, if we set $\eta=1$, the spike growth is clearly observed in the interval of the grazing angle from 2.5 till 5 degrees. In particular, decrease in the grazing angle from 3 to 2 degrees results in a strong suppression of the spike growth, its height being decreased by a factor of 2 . This effect was experimentally observed in $[1]$.

Explanation of non-linear dependence of spike growth on the grazing angle consists in the following. In the limiting case of extremely small grazing angle of an incoming beam $\theta$, the parameter $U<<1$ and, hence, the argument of the logarithmic function in Eq. (8) is equal approximately to $1+k U h_{0}$. Then we obtain that $\Delta h=h(\rho)-\bar{h} \approx h_{0}(\rho)$, i.e. the spike growth is not observed at all. This case is characterized by small polymerization probabilities (2)-(3), so that the right side of Eq. (5) is very small and thus the growth of spike is very slow.

The same conclusion is valid in the opposite limiting case of large grazing angle $\theta$, when $U>>1$, while $U V<<1$ because of exponentially small multiplayer $\exp \left(-\bar{N} \eta|t|^{2}\right)$. Then the argument of logarithmic function in Eq. (8) is close to $\exp \left(k U h_{0}\right)$ resulting once again in $\Delta h=h(\rho)-\bar{h} \approx h_{0}(\rho)$. The physical reason of the phenomenon is explained as follow. The field intensity on a flat surface is high in the case of large grazing angle (see Fig. 1), so that the polymerization probability achieves $P_{0} \sim 80-90 \%$ even for molecule falling onto a flat surface, the value being only slightly less as compared with that at the top of the feature of the $1.5 \mathrm{~nm}$ height. Hence, the right side of Eq. (5) is small as in the previous case and the surface growth occurs almost uniformly in average, because the probability of desorption is almost the same independently of the point on the surface, whether it is placed on the feature top or on the average surface.

Figure $2 \mathrm{~b}$ demonstrates one more interesting feature of spike growth. If the grazing angle of incident radiation is fixed, the spike growth is observed in a limited, from above and below, interval of the radiation flux density. If we set $\theta=3^{\circ}$, 
the spike height achieves maximal value at the parameter $\eta \sim 1$. Decreasing or increasing radiation flux density by a factor of 10 results in the total disappearing of spikes growth. Disappearing of spikes at very high radiation flux density is explained by the same reason as in the case of large grazing angles: the probability of polymerization of hydrocarbon molecules is almost the same independently of the point on the surface, whether it is placed on the feature top or on the average surface. The last effect was not predicted in the previous our work [1], while it follows directly from Eq. (8).

Let consider now high enough radiation flux density, so that, according to Fig. $2 b$, the spike height achieves maximal value at the grazing angles $\theta$ much smaller than the critical angle $\theta_{\mathrm{c}}$ of TER. Then $\operatorname{Im} r(\theta) \approx-2 \sin \theta / \sin \theta_{c}$ and $|t(\theta)|^{2} \approx 4 \sin ^{2} \theta / \sin ^{2} \theta_{c}$, and, hence, the parameter $U(\theta)$ and $V(\theta)$ in Eq. (8) depends only on the product $\eta \sin ^{2} \theta$ rather than on the values of $\eta$ and $\theta$ separately. Dependence of the spike height on the parameter $\eta \sin ^{2} \theta$ is shown in Fig. 8 for different radiation flux density (parameter $\eta=1$ or 10 ) and diffusion parameter $\xi=3$ or 6 . As seen, dependence of spike height on $\eta \sin ^{2} \theta$ represents universal curve at the fixed $\xi$, while the curves are slightly shifted in respect to each other with varying parameter $\xi$. The spike height is maximal at $\eta \sin ^{2} \theta \sim 0.004-0.006$, what corresponds to the polymerization probability of hydrocarbon molecule at a flat surface of about $P_{0} \sim 15-20 \%$. This value is optimal for the spike growth and, hence, represents the worst situation for operation of mirrors in FEL beamlines. Next, if we observe intensive growth of spikes at a certain grazing angle $\theta$, then decreasing (attenuating) or increasing (focusing) radiation flux density by, say, a factor of 4 will increase or decrease the "worst" for the practice value of the grazing incidence angle by a factor of 2 . This conclusion is independent on the values of the free parameters of our model, which thus can be justified or rejected basing on experimental observations.

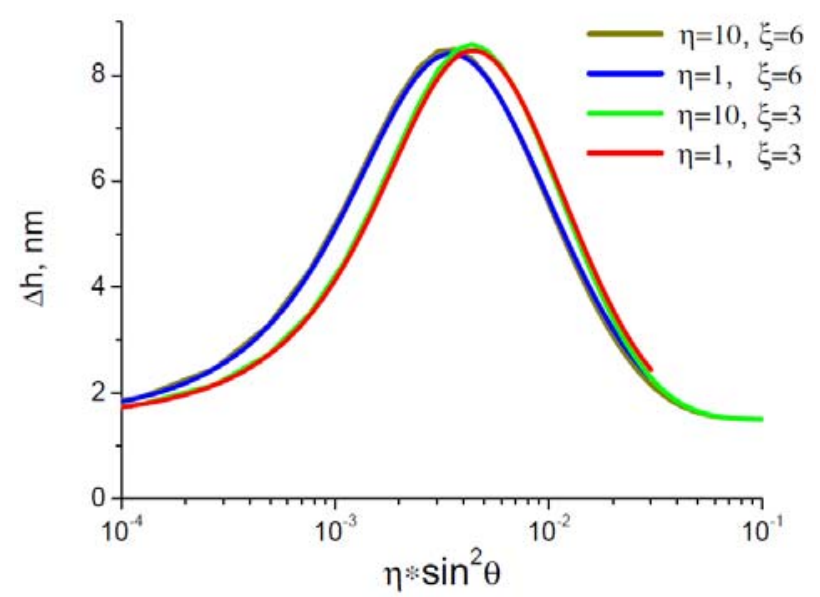

Fig.8. Dependence of the spike height on the parameter $\eta \sin ^{2} \theta$. Calculations were performed for different radiation flux density (parameter $\eta=1$ or 10) and diffusion parameter $\xi=3$ or 6 . The feature height on a virgin substrate was $1.5 \mathrm{~nm}$ and the averaged carbonaceous film thickness is equal to $\bar{h}=15 \mathrm{~nm}$ at $\xi=6$, while $\bar{h}=20.5 \mathrm{~nm}$ at $\xi=3$ to provide the same maximal spike height.

\section{REFERENCES}

[1] Kozhevnikov, I.V., Buzmakov, A.V., Siewert, F., Tiedke, K., Störmer, M., Samoylova, L. and Sinn, H, "Growth of nano-dots on the grazing incidence mirror surface under FEL irradiation", J. Synchr. Rad., 23 78-90 (2016).

[2] Tiedtke, K., Azima, A., von Bargen, N., Bittner, L., Bonfigt, S., Duesterer, S., Faatz, B., Fruehling, U., Gensch, M., Gerth, Ch., Guerassimova, N., Hahn, U., Hans, T., Hesse, M., Honkavaara, K., Jastrow, U., Juranic, P., Kapitzki, S., Keitel, B., Kracht, T., Kuhlmann, M., Li, W.B., Martins, M., Nunez, T., Ploenjes, E., Redlin, H., Saldin, E.L., Schneidmiller, E.A., Schneider, J.R., Schreiber, S., Stojanovic, N., Tavella, F., Toleikis, S., Treusch, R., Weigelt, H., Wellhoefer, M., Wabnitz, H., Yurkov, M.V. and Feldhaus, J., "The soft X-ray freeelectron laser FLASH at DESY: beamlines, diagnostics and end-stations", New J. Phys., 11023029 (2009). 
[3] Boller, K., Haelbich, R.-P., Hogrefe, H., Jark, W. and Kunz, C., "Invesigation of carbon contamination of mirror surfaces exposed to synchrotron radiation", Nucl. Instrum. Methods, 208, 273-279 (2008).

[4] Hollendshead, J. and Klebanoff, L., "Modeling radiation-induced carbon contamination of extreme ultrviolet optics", J. Vac. Sci. Technol., 24, 64-82 (2006).

[5] Family, F. and Viscek, T., "Scaling of the active zone in the Eden process on percolation networks and the ballistic deposition model", J. Phys. A, 18, L75-L82 (1985).

[6] Family, F., "Scaling of rough surfaces: effects of surface diffusion", J. Phys. A, 19, L441-L446 (1986). 